\title{
Effects of telmisartan on serum adiponectin and left ventricular remodeling in patients with essential hypertension.
}

\author{
Shu-Po Lin ${ }^{1}$, Yong-Tang Shi ${ }^{1}$, Qing-Min Wei ${ }^{1}$, Chun-Gui Zheng ${ }^{2}$, Xiao-Peng Tian ${ }^{\text {* }}$ \\ ${ }^{1}$ Department of Cardiology, the People's Hospital of Xingtai, Xingtai, PR China \\ ${ }^{2}$ Department of Nursing, Xingtai Medical College, Xingtai, PR China \\ ${ }^{3}$ Department of Internal Medicine, the People's Hospital of Xingtai, Xingtai, PR China
}

\begin{abstract}
The aim of this study was to observe the changes in the serum Adiponectin (APN) levels and Left Ventricular Hypertrophy (LVH) in hypertensive patients before and after telmisartan treatment. Two hundred and forty hypertensive patients complicated with LVH were divided into a telmisartan group and an amlodipine group. The changes of these patients' blood pressure, APN, and Left Ventricular Mass index (LVMI) treatment before and $12 \mathrm{w}$ after treatment were compared. The systolic and diastolic blood pressures in both groups were significantly lower than those before treatment $(\mathbf{P}<0.01)$. The difference in diastolic blood pressures between the two groups was not statistically significant $(P>0.05)$. Serum APN levels in the telmisartan group were significantly higher than those before treatment $(P<0.01)$, while LVMI was significantly lower than before $(P<0.01)$. Serum APN levels and LVMI had no significant changes in the amlodipine group before and after treatment $(P>0.05)$. Compared with amlodipine, telmisartan significantly increased the serum APN levels and significantly reversed the Left Ventricular Remodeling (LVRM) while reducing the blood pressure.
\end{abstract}

Keywords: Telmisartan, Essential hypertension, Adiponectin, Left ventricular remodeling. Accepted on January 30, 2018

\section{Introduction}

Essential hypertension is a common cardiovascular disease. Left Ventricular Remodeling (LVRM) damages the target organs in hypertension and is an independent risk factor for inducing cardiovascular events such as heart failure, myocardial infarction, arrhythmia, and sudden cardiac death [1]. Adiponectin (APN) is a novel adipokine that is produced by white adipocytes and can improve insulin resistance, antiatherosclerosis, and anti-inflammatory effect [2]. In recent years, it has been found [3] that APN is closely related to the onset and progression of hypertensive ventricular remodeling. Telmisartan is an antagonist that competitively inhibits angiotensin II (Ang II) receptor 1, effective in lowering blood pressure, protecting the heart, and reversing hypertensive ventricular remodeling. The influence of telmisartan on APN levels and APN receptor expression in myocardium may be one of the mechanisms of its cardioprotective effect. At present, there have been few clinical studies on the relationship between Left Ventricular Hypertrophy (LVH) and serum APN concentration, as well as the influences of telmisartan on them. This study was intended to further explore the mechanism of left ventricular remodeling and the protective effect of telmisartan reversal in hypertensive patients, so as to provide the basis for clinical treatment.

\section{Materials and Methods}

\section{Clinical data}

Two hundred and forty essential hypertensive patients complicated with LVH were selected from outpatients in the People's Hospital of Xingtai (Address: Xingtai, China) from June 2016 to June 2017 according to the inclusion criteria. Among them, there were 144 males and 96 females aged 50 to $70(62.21 \pm 9.02$ y). Inclusion criteria: The diagnostic criteria of hypertension [4] followed the seventh report of the Joint National Committee on Detection, Evaluation, and Treatment of High Blood Pressure (JNC 8), that is, the Systolic Blood Pressure (SBP) was not less than $140 \mathrm{mmHg}$ and/or the Diastolic Blood Pressure (DBP) was not less than $90 \mathrm{mmHg}$ when anti-hypertensive drugs were not used. In addition, all included candidates did not receive any hypoglycemic, lipidlowering, and weight-losing treatment. Meanwhile, hypertensive patients were treated without any antihypertensive treatment for at least $2 \mathrm{w}$, in the $24 \mathrm{~h}$ before and during the study, their diet routines should remain stable. The diagnostic criteria of LVH [5]: Left Ventricular Mass Index (LVMI) greater than $125 \mathrm{~g} / \mathrm{m}^{2}$ for a male or greater than 120 $\mathrm{g} / \mathrm{m}^{2}$ for a female. Exclusion criteria: Those with secondary hypertension, coronary heart disease, diabetes, New York heart 
function assessment (NYHA): class III and class IV, severe liver dysfunction, acute or chronic infections, tumor, recent surgery or trauma, connective tissue disease, contraindications or allergies to Angiotensin Receptor Blockers (ARBs) and Calcium Channel Blockers (CCBs), and smoking habit. This study was conducted in accordance with the declaration of Helsinki and with the approval from the Ethics Committee of the people's hospital of Xingtai. Written informed consents were obtained from all participants.

\section{Treatment methods}

The patients were randomly divided into a telmisartan group and an amlodipine group. The former was orally given telmisartan (Micardis ${ }^{\circledR}$, Boehringer Ingelheim Pharma GmbH \& Co.KG) of $40 \mathrm{mg}$, qd, while the latter was orally given amlodipine (Norvasc ${ }^{\circledR}$, Pfizer) of $5 \mathrm{mg}$, qd. The patients' APN and LVMI were repeatedly measured before and $12 \mathrm{w}$ after treatment. The drug efficacy and safety were followed up via outpatient consultation and telephone calls. Throughout the trial, the patients did not take any other antihypertensive drugs, hypolipidemic drugs, tricyclic antidepressants, or long-acting nitrate drugs, and the approach of administration remained unchanged.

\section{Measurement indicators}

APN measurement: Upon waking up in the morning, $5 \mathrm{ml}$ of venous blood was collected and placed in a dry tube, which was centrifuged at $4000 \mathrm{r} / \mathrm{min}$ for $5 \mathrm{~min}$ and reserved in a refrigerator at a temperature of $-80^{\circ} \mathrm{C}$, as specified by the kit instructions. The radioimmunoassay kit for serum APN was supplied by Linco Research Inc., USA, with an intra-assay difference of $<6.25 \%$ and an inter-assay difference of $<9.25 \%$. Philips iE33 Color Doppler System was employed to measure and calculate LVMI. The measurement was performed by the same experienced ultrasound operator.

\section{Statistical analysis}

Categorical variables are presented as frequencies, Continuous variables are presented as the mean and standard deviation or the median and quartiles. Normal analysis was performed between the two groups, while t-test was adopted to compare normal distributed variables. Mann-Whitney $U$ test was adopted to compare non-normal distributed variables. The ratios were compared by using chi-square test. Wilcoxon Signed Rank Test was used to compare the same group before and after medication. Linear correlation analysis was applied for the correlation between serum APN and LVMI. A twosided $\mathrm{P}$ value $<0.05$ was considered statistically significant. SPSS 21.0 software was used for statistical analysis.

\section{Results}

\section{Baseline data comparison}

A total of 240 patients met the criteria for inclusion and exclusion. However, since two cases were lost to follow-up in both groups, 236 patients were included in the statistical treatment. Before treatment, the differences in baseline levels, such as age, Body Mass Index (BMI), duration of hypertension, blood pressure, blood glucose, blood lipids, and heart rate, between the two groups were not statistically significant $(\mathrm{P}>0.05)$, as shown in Table 1 .

Table 1. Comparison of baseline data between two groups $(\bar{x} \pm s)$.

\begin{tabular}{llll}
\hline Baseline data & Telmisartan group & Amlodipine group & P value \\
\hline Male/Female (case) & $74 / 44$ & $68 / 50$ & 0.494 \\
\hline Age $(\mathrm{y})$ & $62.12 \pm 8.56$ & $62.35 \pm 7.56$ & 0.424 \\
\hline BMI $\left(\mathrm{kg} / \mathrm{m}^{2}\right)$ & $25.43 \pm 2.01$ & $24.83 \pm 3.01$ & 0.301 \\
\hline Duration (y) & $9.58 \pm 5.32$ & $10.11 \pm 4.92$ & 0.495 \\
\hline SBP (mmHg) & $158 \pm 14$ & $156 \pm 15$ & 0.378 \\
\hline DBP (mmHg) & $88 \pm 10$ & $87 \pm 10$ & 0.475 \\
\hline HR (times/min) & $70.25 \pm 6.31$ & $72.12 \pm 5.83$ & 0.394 \\
\hline Blood glucose (mmol/L) & $6.05 \pm 0.64$ & $6.12 \pm 0.58$ & 0.303 \\
\hline TC (mmol/L) & $5.28 \pm 0.95$ & $5.46 \pm 1.10$ & 0.366 \\
\hline TG (mmol/L) & $1.69 \pm 0.73$ & $1.92 \pm 0.89$ & 0.463 \\
\hline HDL-C (mmol/L) & $1.32 \pm 0.38$ & $1.35 \pm 0.34$ & 0.420 \\
\hline LDL-C (mmol/L) & $2.69 \pm 0,67$ & $2.63 \pm 0.84$ & 0.494 \\
\hline
\end{tabular}

\section{Changes in blood pressure}

After treatment, SBP and DBP were significantly lower than before treatment in both groups, and the differences were statistically significant $(\mathrm{P}<0.05)$. However, the decrease of SBP in the telmisartan group was less than that of the amlodipine group, with statistically significant differences $(\mathrm{P}<0.05)$. The difference in DBP between the two groups was not statistically significant $(\mathrm{P}>0.05)$, as shown in Table 2 .

Table 2. Changes in blood pressure of two groups before and after drug treatment.

\begin{tabular}{lllc}
\hline Blood pressure & \multicolumn{1}{c}{ Telmisartan group } & Amlodipine group \\
\cline { 2 - 4 } & Before treatment & After treatment & Before treatment \\
\hline SBP $(\mathrm{mmHg})$ & $158 \pm 14$ & $135 \pm 8$ & $156 \pm 15$ \\
\hline $\mathrm{DBP}(\mathrm{mmHg})$ & $88 \pm 10$ & $76 \pm 7$ & $87 \pm 10$ \\
\hline
\end{tabular}




\section{Changes in APN and LVMI}

Before treatment, serum APN levels in both groups were significantly lower than the normal values, while the LVMI was higher than normal, but the difference between groups was not statistically significant. After treatment, in the telmisartan group, serum APN levels were significantly higher than before treatment, with a statistically significant difference $(\mathrm{P}<0.05)$, while LVMI was significantly lower, with a statistically significant difference $(\mathrm{P}<0.01)$. However, in the amlodipine group, the difference in APN levels was not statistically significant $(\mathrm{P}=0.81>0.05)$ and LVMI had no significant changes before after treatment. Compared with the amlodipine group, APN levels showed statistically significant difference $(\mathrm{P}<0.01)$ and LVMI was significantly improved in the telmisartan group after treatment, with statistically significant differences $(\mathrm{P}<0.01)$, as shown in Table 3 .

Table 3. Changes in APN and LVMI in both groups before after treatment.

\begin{tabular}{lllll}
\hline Items & \multicolumn{2}{l}{ Telmisartan group } & \multicolumn{2}{l}{ Amlodipine group } \\
\cline { 2 - 5 } & $\begin{array}{l}\text { Before } \\
\text { treatment }\end{array}$ & $\begin{array}{l}\text { After } \\
\text { treatment }\end{array}$ & $\begin{array}{l}\text { Before } \\
\text { treatment }\end{array}$ & $\begin{array}{l}\text { After } \\
\text { treatment }\end{array}$ \\
\hline APN $(\mu \mathrm{g} / \mathrm{ml})$ & $6.88 \pm 1.94$ & $7.58 \pm 2.09$ & $6.69 \pm 1.89$ & $6.72 \pm 1.92$ \\
\hline LVMI $\left(\mathrm{g} / \mathrm{m}^{2}\right)$ & $134.18 \pm 6.85$ & $110 \pm 5.24$ & $132.88 \pm 6.78$ & $130.76 \pm 7.39$ \\
\hline
\end{tabular}

\section{Correlation between LVMI and serum APN}

Linear correlation analysis was performed on the LVMI and serum APN in hypertensive patients complicated with LVH before after treatment. Results showed a linear correlation between LVMI and serum APN. Pearson correlation was used to test the correlation between LVMI and serum APN. $(\mathrm{r}=-0.386, \mathrm{P}<0.05)$, as shown in Graph 1 .

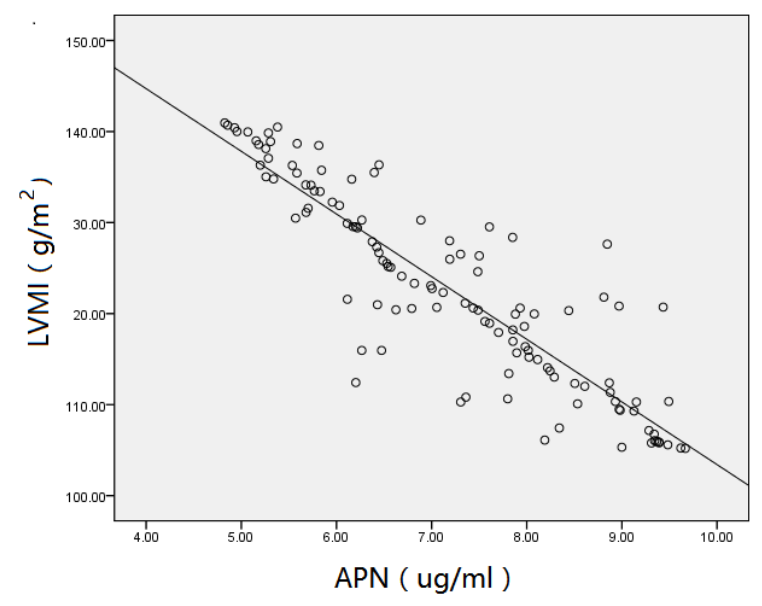

Graph 1. The Scaterplot graph for correlation between LVMI and serum APN.

\section{Discussion}

Left ventricular remodeling is the most common complication of hypertension and an independent risk factor for inducing cardiovascular events such as heart failure, myocardial infarction, arrhythmia, and sudden cardiac death [6,7]. Moreover, it is the pathophysiological basis for heart dysfunction and failure. Ventricular remodeling is not only an adaptive response to hemodynamic disorders, as well as to changes in neurohumoral fluid and endocrine factors, in hypertension, but also an important pathological basis for the worsening of hypertension and abnormal cardiac function. Therefore, it is of great significance to prevent and reverse myocardial remodeling in managing heart failure and improving the prognosis of patients.

Adiponectin (APN) is a novel adipokine that is produced by white adipocytes and can regulate the body's glycolipid metabolism, while improving insulin resistance, antiatherosclerosis, and anti-inflammatory effects. Recently, APN has been found to play a role in the clinical progression of essential hypertension and left ventricular remodeling. Hong et al. [8] found that the decreased levels of APN were closely related to the diastolic dysfunction caused by LVH. Mitsuhashi et al. [9] used ECG to evaluate LVH and discovered that low APN was an independent risk factor for LVH. Kozakova et al. [3] investigated the normal population and also found that the circulating APN was independent of age and metabolic factors but was negatively correlated with left ventricular volume (standardized $\mathrm{r}=-0.28, \mathrm{P}<0.05$ ) and ventricular wall thickness (standardized $\mathrm{r}=-0.27, \mathrm{P}<0.01$ ). In the latest study published in Nature Medicine, Shibata et al. suggested that in APN knockout mice, cardiac hypertrophy could be easily induced by pressure load, increasing mortality rate. All of these findings are associated with the increased Extracellular SignalRegulated Kinase (ERK) and decreased AMPK signaling in the myocardium. After the APN gene carried by adenovirus reenters the body, such trend can be reversed. Fujita et al. [10] investigated the APN and PPAR-alpha knockout mice and also found that APN could inhibit AngII-induced myocardial fibrosis and thus cardiac remodeling by activating the AMPKdependent PPAR-alpha. Recently, Xie et al. [11] found that fibroblasts were highly expressed in rats with APN and AdipoR1 cultured in vitro, and that poly (ADP-ribose) polymerase-1 (PARP-1) could inhibit the expressions of APN and AdipoR1 by acting on PPARa, leading to fibroblast proliferation. This study also found that the serum APN levels decreased significantly in essential hypertension complicated with ventricular remodeling, which was consistent with the above findings.

Telmisartan is a novel angiotensin receptor blocker of nonpeptide benzimidazoles. It competitively inhibits the AT1 receptors to block the RASS system. Studies have shown that telmisartan provides cardiovascular protection similar to that of ACEI drugs while lowering the blood pressure, especially in the prevention and reversal of ventricular remodeling caused by hypertension [12]. At present, research on its cardiovascular protection mainly focuses on its role in the RASS system [13]. In this study, it was found that serum APN levels were lower than normal in hypertension and hypertensive ventricular remodeling. In this regard, telmisartan was used to treat hypertensive patients. Results showed that telmisartan could result in increased levels of serum APN while lowering blood 
pressure, protection the heart, and reversing myocardial remodeling. But the underlying mechanisms remained unclear. A possible process could be as follows: Telmisartan reverses ventricular remodeling by increasing the APN levels and upregulating the myocardial expression of APN receptors, so as to activate the intracellular signaling pathways, such as AMPK pathway [14], and to inhibit the phosphorylation of ERK [15]. By doing so, it can improve the myocardial energy metabolism and inhibit cardiac hypertrophy and interstitial cell proliferation, thereby inhibiting and reversing the hypertensive ventricular remodeling. However, there have been few studies on this topic, and more preliminary research is needed.

\section{References}

1. Levy D, Garrison RJ, Savage DD, Kannel WB, Castelli WP. Prognostic implications of echocardiographically determined left ventricular mass in the Framingham Heart Study. N Engl J Med 1990; 322: 1561-1563.

2. Mantzoros CS, Li T, Manson JE, Meigs JB, Hu FB. Circulating adiponectin levels are associated with better glycemic control, more favorable lipid profile, and reduced inflammation in women with type 2 diabetes. J Clin Endocrinol Metab 2005; 90: 4542-4545.

3. Hong SJ, Park CG, Seo HS, Oh DJ, Ro YM. Associations among plasma adiponectin, hypertension, left ventricular diastolic function and left ventricular mass index. Blood Press 2004; 236-242.

4. James PA, Oparil S, Carter BL. 2014 Evidence-based guideline for the management of high blood pressure in adults: Report from the panel members appointed to the Eighth Joint National Committee (JNC 8). JAMA 2014; 311: 507-520.

5. Elias MF, Sullivan LM, Elias PK, DAgostino RB Sr, Wolf PA, Seshadri S, Au R, Benjamin EJ, Vasan RS. Left ventricular mass, blood pressure, and lowered cognitive performance in the Framingham offspring. Hypertension 2007; 49: 439-442.

6. Daniels SD, Meyer RA, Loggie JM. Determinants of cardiac involvement in children and adolescents with essential hypertension. Circulation 1990; 82: 1243.

7. Schillaci G, Verdecchia P, Porcellati C, Cuccurullo O, Cosco C, Perticone F. Continuous relation between left ventricular mass and cardiovascular risk in essential hypertension. Hypertension 2000; 35: 580-586.

8. Hong SJ, Park CG, Seo HS, Oh DJ, Ro YM. Associations among plasma adiponectin, hypertension, left ventricular diastolic function and left ventricular mass index. Blood Press 2004; 13: 236-242.

9. Mitsuhashi H, Yatsuya H, Tamakoshi K, Matsushita K, Otsuka R, Wada K, Sugiura K, Takefuji S, Hotta Y, Kondo T, Murohara T, Toyoshima H. Adiponectin level and left ventricular hypertrophy in Japanese men. Hypertension 2007; 49: 1448-1454.

10. Fujita K, Maeda N, Sonoda M, Ohashi K, Hibuse T, Nishizawa H, Nishida M, Hiuge A, Kurata A, Kihara S, Shimomura I, Funahashi T. Adiponectin protects against angiotensin II-induced cardiac fibrosis through activation of PPAR-alpha. Arterioscler Thromb Vasc Biol 2008; 28: 863-870.

11. Xie H, Huang D, Zhang S, Hu X, Guo J, Wang Z, Zhou G. Relationships between adiponectin and matrix metalloproteinase-9 (MMP-9) serum levels and postoperative cognitive dysfunction in elderly patients after general anesthesia. Aging Clin Exp Res 2016; 28: 1075-1079.

12. Galzerano D, Tammaro P, del Viscovo L, Lama D, Galzerano A, Breglio R, Tuccillo B, Paolisso G, Capogrosso P. Multicentre randomized study on the effect of telmisartan compared to carvedilol on LV mass: three dimensional echocardographic and magnetic resonance assessment. J Hypertens 2005; 22: 112.

13. Kondo K, Toh R, Ishida T. Comparison of telmisartan/ amlodipine and telmisartan/hydrochlorothiazide in the treatment of Japanese patients with uncontrolled hypertension: the TAT-Kobe study. Blood Press Monit 2016; 21: 171-177.

14. Daskalopoulos EP, Dufeys C, Bertrand L, Beauloye C, Horman S. AMPK in cardiac fibrosis and repair: Actions beyond metabolic regulation. J Mol Cell Cardiol 2016; 91: 188-200.

15. Wang Y, Zhang J, Zhang L, Gao, Wu X. Adiponectin attenuates high glucose-induced apoptosis through the AMPK/p38 MAPK signaling pathway in NRK-52E cells. PLoS One 2017; 12: 1001-1004.

\section{*Correspondence to}

Xiao-Peng Tian

Department of Internal Medicine

The People's Hospital of Xingtai

PR China 